\title{
Short-term wind power forecasting using long-short term memory based recurrent neural network model and variable selection
}

\author{
Umit Cali*, Vinayak Sharma \\ University of North Carolina at Charlotte, Charlotte, NC, USA
}

\begin{abstract}
Increasing concern for the environment has led to governments and companies pushing for renewable power generation. The share of wind power for the supply of electricity has been increasing during the last two decades. Increased penetration levels of intermittent power sources like wind power became a challenge for the power companies. Therefore, the need to make accurate forecasts for wind power generation has become a very critical issue for the power system operators. This paper presents a novel approach to forecasting 1 to 24 hours ahead wind power using Long Short-Term Memory based Recurrent Neural Network (LSTM-RNN). The model is based on knowledge from the data, that both weather and wind power have short-term temporal dependencies. The proposed model is implemented using historical generated wind power and Numerical Weather Prediction (NWP) data for Sotavento, a wind farm in Spain. Input parameters from the NWP data are selected by performing a sensitivity analysis for variable selection technique.
\end{abstract}

Keywords: LSTM-RNN, wind power forecasting, renewable energy, machine learning, neural network.

\section{Introduction}

A major part of the World's energy need is fulfilled by burning fossil fuels. However, burning fossil fuels like coal, oil and gas to produce energy releases a large number of greenhouse gasses, which are a major concern for the environment. [1]. One way of producing clean and carbon-free energy is to use renewable sources of energy like wind and solar power to meet the increasing demand for energy. Wind power is one of the fastest growing sources of power generation, with countries like China, United States, India and Germany moving towards wind energy for their energy needs [2]. The intermittency of the power output characteristics of wind power resources became one of the biggest challenges for the power system operators [3]. The energy produced by wind depends heavily on weather variables such as wind speed, wind direction, temperature, etc. Since the weather cannot be controlled, the amount of energy produced by wind cannot be controlled as well. This makes the integration of wind power to the grid a major challenge. To overcome this issue, it is important to make accurate wind energy forecasts in order to reduce the uncertainty in the amount of energy that is going to be produced in future.

Over the years various tools and methodologies have been developed to forecast wind power. Physical methods that make use of atmospheric descriptions to forecast wind power have been used[4]. A common approach to forecasting wind power is to use numerical weather prediction (NWP) data in statistical forecasting models[5]. Statistical models such as polynomial regression[6], ARMA[7] and ARIMA [8, 9] have been extensively reported in the literature for wind power forecasting. However, with the development of intelligent machine learning algorithms like neural networks and support vector machines (SVM) for time series forecasting, these were used to forecast wind power as well. Machine learning techniques such as artificial neural network (ANN) and SVM are capable of performing complex

\footnotetext{
* Manuscript received July 26, 2018; revised January 4, 2019.

Corresponding author. Tel.: 001+9802593583; E-mail address: ucali@uncc.edu

doi: 10.12720 /sgce.8.2.103-110
} 
nonlinear modelling for a large amount of data making it suitable for time series forecasting [10]. In [11], wind speed wind direction and wind turbine parameters are used to forecast wind power using a neural network. Feed-forward ANN is used in [12], to predict one day ahead wind power.

Conventional neural network based deterministic wind power forecasting models lack the ability to incorporate previous information about wind power. This can be solved using recurrent neural networks (RNN). RNN based forecasting models have been used to forecast wind power[13] [14] . In [15], a RNN model is used to predict the uncertainty in wind power. In [16], long-term predictions are made using a RNN based forecasting model. A special kind of RNN model called as long short-term memory (LSTM) RNN is used to predict stock prices in [17]. In [18], an LSTM-RNN with 50 neurons in the LSTM layer is used to predict the life of lithium-ion batteries. LSTM-RNN has been explored in various fields such as speech recognition [19] and handwriting recognition [20], but very little literature could be found on the use of LSTM-RNN in renewable energy forecasting [21].

The main aim of the present research is to propose a novel LSTM-RNN based model for 1 to 24 hours ahead wind power forecasting. The input parameters chosen for the LSTM-RNN model are selected using a sensitivity analysis test. Results show that the LSTM-RNN model performs well and gives accurate results for short-term wind power forecasts.

\section{Forecasting Methodology}

Machine learning algorithms are used to predict wind power using weather inputs such as wind speed, wind direction, temperature, etc. The future value of wind power depends on weather variables, but it also depends on the previous value of the wind power generated. The amount of wind power being produced in the current hour influences the amount of wind power generation in the next hour.

Recurrent Neural Networks (RNN) are used to incorporate this short-term temporal dependency in wind power. RNN can consider previous information and make a forecast based on that. Fig 1 shows a basic RNN architecture. How far in the past does the model need to look is decided by analyzing the autocorrelation function. But, in case of time series forecasting, the state of the model at the just previous timestep is not enough. The value of the next timestep may depend on more than one previous timesteps. Wind power has a short-term as well as a long-term temporal dependency. A simple RNN model is not able to cope with long-term time dependencies. The long-term information gets lost due to vanishing gradient problem in case of a simple RNNs [22]. This is solved using LSTM based RNN.



Fig. 1. Description of the architecture of a RNN.

LSTM-RNN maps input variables to the output variables using a dynamic structure with the addition of LSTM cells. In order to overcome the lack of ability of the simple RNN model to deal with long-term dependencies, the LSTM-RNN consists of a memory cell that stores these long-term dependencies. In 
addition to the memory cell, the LSTM cell contains input, output and forget cells that facilitate the reading and writing to and from the memory cell. The following equations are used to explain the various components of the LSTM cell:

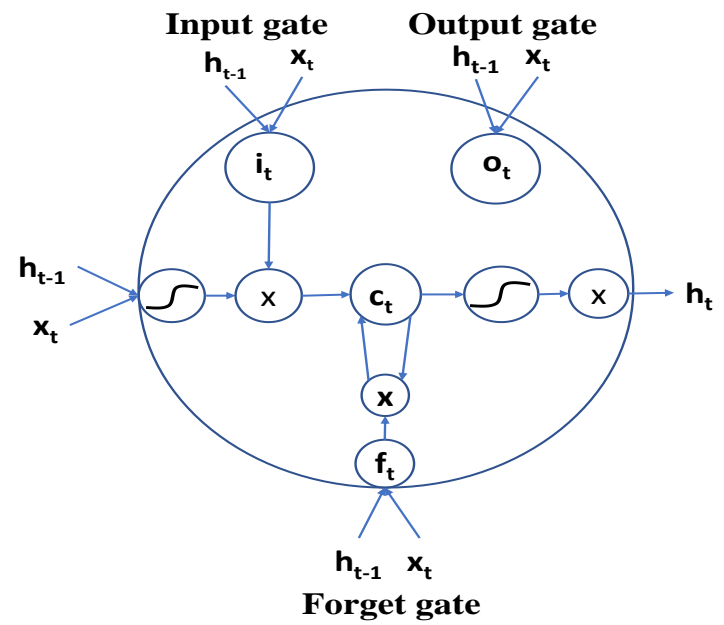

Fig.2. Description of LSTM cell configuration.

$\mathrm{i}_{\mathrm{t}}=\mathrm{S}\left(\mathrm{w}_{\mathrm{xi}} \mathrm{x}_{\mathrm{t}}+\mathrm{w}_{\mathrm{hi}} \mathrm{h}_{\mathrm{t}-1}+\mathrm{b}_{\mathrm{i}}\right)$,

$\mathrm{o}_{\mathrm{t}}=\mathrm{S}\left(\mathrm{w}_{\mathrm{xo}} \mathrm{x}_{\mathrm{t}}+\mathrm{w}_{\mathrm{ho}} \mathrm{h}_{\mathrm{t}-1}+\mathrm{b}_{\mathrm{o}}\right)$,

$f_{t}=S\left(w_{x f} x_{t}+w_{h f} h_{t-1}+b_{f}\right)$,

$c_{t}=c_{t-1} f_{t}+i_{t} \tanh \left(w_{x c} x_{t}+w_{h c} h_{t-1}+b_{c}\right)$,

$h_{t}=o_{t} \tanh \left(c_{t}\right)$.

where, $S$ is the sigmoid function, $w$ is the weight at each gate, $w_{12}$ is the weight between gates 1 and $2, b$ is the bias at each gate, $i$ is the input gate, $o$ is the output gate, $f$ is the forget gate and $c$ is the memory cell. Fig 2 shows the configuration of an LSTM memory cell where $i_{t}$ is the input at timestep $t$ and $h_{t-1}$ is the state of the hidden layer at timestep $t-1$.

\section{Data Mining Techniques}

\subsection{Description of the used data}

The data used for the model comes from Sotavento, a wind farm located in Galicia, Spain. The wind farm consists of 24 wind turbines with a power rating of $17.56 \mathrm{MW}$ and an annual generation of about $33000 \mathrm{MWh}$. One year of data from $1^{\text {st }}$ January 2016 to $31^{\text {st }}$ December 2016 with an hourly resolution with a total of 8757 data points is used. The NWP data for the location comes from Weather \& Energy PROGnoses (WEPROG). The NWP data contains 75 members with wind speeds at 10m, 75m, $100 \mathrm{~m}$ and $170 \mathrm{~m}$ height each, wind direction at $10 \mathrm{~m}$ and $170 \mathrm{~m}$ height, temperature, pressure, etc.

\subsection{1to 24 hours ahead LSTM-RNN based model}

The performance of the wind power forecasts is highly reliant on the forecast horizon. Due to temporal dependencies, the accuracy of the forecast is more for few hours ahead and less for several hours ahead. The forecasts for the first hour is based on the measured power from the previous hour. However, for the 
subsequent hours, the measured value of the previous hour is absent, so the forecast value of the previous hour is used. The forecast value has some error associated with it, for several hour-ahead forecasts, this error gets accumulated and the accuracy decreases.

Autocorrelation function of the historical wind power time series was calculated to understand the number of lags required for the model. From Fig 3, it is observed that after 24 lags the autocorrelation value drops below 0.4. For this reason, 24 lags were considered for the models. The number of epochs and the number of hidden layers and neurons were decided upon after conducting various test experiments and the best results were chosen to build the models. The most accurate results were found with one hidden layer and 50 hidden neurons. In this paper, wind power forecasting is investigated for forecasting horizons from 1 hour ahead to 24 hours ahead using LSTM-RNN model described above.

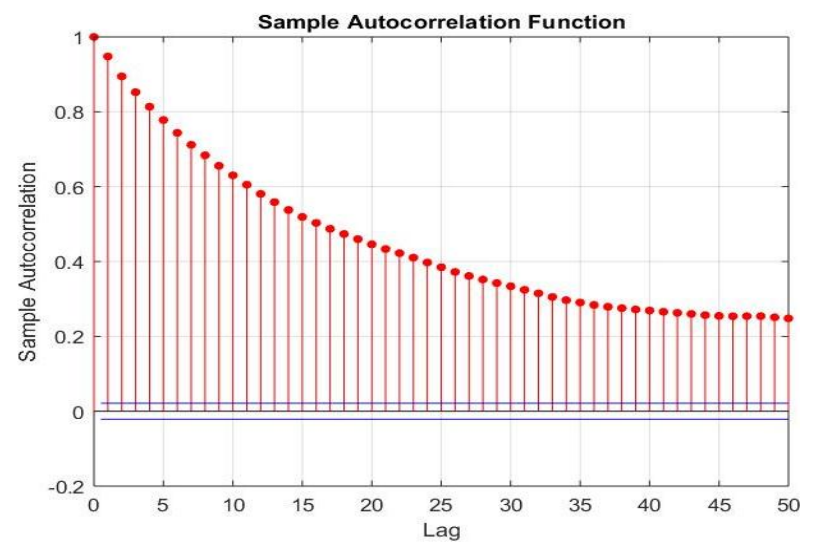

Fig. 3. The autocorrelation plot.

\subsection{Sensitivity analysis for variable selection}

Wind power generation is highly influenced by weather variables. Some variables can contribute positively to the accuracy of the model while others can have a negative impact. In this experiment, a sensitivity analysis is performed to select the most influential variables to get the most accurate model for wind power forecasting. An LSTM-RNN model with one hidden layer consisting of 50 hidden neurons is used to predict one day-ahead wind power. 9 months of the data, from $1^{\text {st }}$ January to $30^{\text {th }}$ September is used for training the model and 3 months of the data from $1^{\text {st }}$ October to $31^{\text {st }}$ December is used for testing the model's performance. Nine forecasting models (F1-F9) are created using different combinations of input variables. Table 1 shows the different input combinations used for the experiments. The experiment F9 contains all the available inputs from the NWP data.

Table 1. Description of the input combinations for sensitivity analysis

\begin{tabular}{|c|c|c|c|c|c|c|c|c|}
\hline PARAMETERS/EXPERIMENTS & $F 1$ & $F 2$ & $\overline{F 3}$ & F4 & F5 & F6 & F7 & $\overline{F 8}$ \\
\hline SURFACE PRESSURE & $\mathrm{x}$ & & & & & & & \\
\hline TEMPERATURE & $\mathrm{x}$ & $\mathrm{x}$ & & & & & $\mathrm{x}$ & $\mathrm{x}$ \\
\hline WIND SPEED AT 10M & $\mathrm{x}$ & $\mathrm{x}$ & $\mathrm{x}$ & $\mathrm{x}$ & & & & $\mathrm{x}$ \\
\hline WIND SPEED AT $35 \mathrm{M}$ & $\mathrm{x}$ & $\mathrm{x}$ & $\mathrm{x}$ & $\mathrm{x}$ & $\mathrm{x}$ & & & $\mathrm{x}$ \\
\hline WIND SPEED AT 100M & $\mathrm{x}$ & $\mathrm{x}$ & $\mathrm{x}$ & $\mathrm{x}$ & $\mathrm{x}$ & $\mathrm{x}$ & $\mathrm{x}$ & $\mathrm{x}$ \\
\hline WIND SPEED AT 170M & $\mathrm{x}$ & $\mathrm{x}$ & $\mathrm{x}$ & & & & & $\mathrm{x}$ \\
\hline WIND DIRECTION AT 35M & $\mathrm{x}$ & $\mathrm{x}$ & $\mathrm{x}$ & $\mathrm{x}$ & $\mathrm{x}$ & $\mathrm{x}$ & $\mathrm{x}$ & \\
\hline WIND DIRECTION AT $170 \mathrm{M}$ & $\mathrm{x}$ & $\mathrm{x}$ & $\mathrm{x}$ & $\mathrm{x}$ & $\mathrm{x}$ & $\mathrm{x}$ & $\mathrm{x}$ & $\mathrm{x}$ \\
\hline
\end{tabular}




\subsection{Validation}

The accuracy of the models is evaluated to see the performance of the models. The performance of the model over the test set is validated using normalized mean absolute error (nMAE), normalized root mean square error (nRMSE). First, the MAE and RMSE are calculated and then divided by the wind farm capacity of $17560 \mathrm{KW}$ to get the normalized validation values. RMSE and MAE are calculated using the following equations:

$$
\begin{gathered}
e[t]=\hat{P}[t]-P[t], \\
R M S E=\sqrt{\frac{1}{T} \sum_{t=1}^{T}(e[t])^{2},} \\
M A E=\frac{1}{T} \sum_{t=1}^{T}|e[t]| .
\end{gathered}
$$

Where $\hat{P}$ is the forecast wind power time series and $P$ is the measured wind power timeseries.

\section{Results}

The nRMSE results of one day ahead wind power forecasts of each experiment (F1 to F9) are presented in Fig 4. Experiment F9 containing all possible 323 inputs has a nRMSE of $12.32 \%$. This implies that more inputs in the model does not mean better accuracy. Experiment F1 containing temperature, surface pressure, wind speeds at $10 \mathrm{~m}, 35 \mathrm{~m}, 100 \mathrm{~m}$ and $170 \mathrm{~m}$ as well as wind direction at $35 \mathrm{~m}$ and $170 \mathrm{~m}$ as inputs has a nRMSE of $10.88 \%$. The experiment F2 containing temperature, wind speeds at $10 \mathrm{~m}, 35 \mathrm{~m}, 100 \mathrm{~m}$ and $170 \mathrm{~m}$ as well as wind direction at $35 \mathrm{~m}$ and $170 \mathrm{~m}$ as inputs has a nRME of $10.80 \%$. This shows that excluding surface pressure increases the accuracy of the model. Temperature is excluded from the inputs in experiment F2 to create experiment F3. It gives a nRMSE of 11.12\%. Experiments F6 and F7 contain wind speed at 100m, wind direction at $35 \mathrm{~m}$ and $170 \mathrm{~m}$. F7 additionally has temperature as another input. Comparing experiments F2 and F3 as well as F6 and F7 shows the importance of temperature as an in. Including temperature increases the accuracy of the forecasts. As it can be seen in Fig 3 that experiment F2 is found to be the most accurate model and is therefore used as the base model in the subsequent experiments for wind power forecasting.

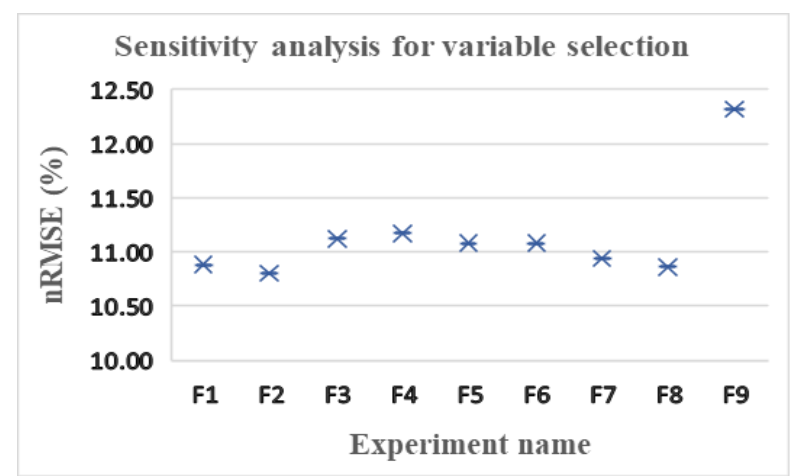

Fig. 4. The results of sensitivity analysis.

Using experiment F2, 1 hour ahead to 24 hour-ahead wind power forecasts were made. Table 2 shows the results obtained from the experiments. It can be observed that the accuracy for one hour ahead wind 
power forecasts is the highest at $4.23 \%$ in terms of nRMSE and $3.01 \%$ in terms of nMAE. As the forecast horizon increases, the accuracy decreases and the nRMSE and nMAE increase.

Table 2. Validation results of 1 to 24 hour ahead forecasts

\begin{tabular}{ccc}
\hline Hours-ahead & nRMSE (\%) & nMAE (\%) \\
\hline $\mathbf{1}$ & 4.23 & 3.01 \\
$\mathbf{2}$ & 4.93 & 3.74 \\
\hline $\mathbf{3}$ & 5.46 & 4.16 \\
\hline $\mathbf{4}$ & 5.59 & 4.25 \\
\hline $\mathbf{5}$ & 5.97 & 4.41 \\
\hline $\mathbf{6}$ & 6.35 & 4.75 \\
\hline $\mathbf{7}$ & 6.65 & 5.06 \\
\hline $\mathbf{8}$ & 7.19 & 5.53 \\
\hline $\mathbf{9}$ & 7.48 & 5.58 \\
\hline $\mathbf{1 0}$ & 7.64 & 5.76 \\
\hline $\mathbf{1 1}$ & 7.99 & 5.83 \\
\hline $\mathbf{1 2}$ & 8.32 & 6.08 \\
\hline $\mathbf{1 5}$ & 9.18 & 6.77 \\
\hline $\mathbf{2 0}$ & 9.96 & 7.51 \\
\hline $\mathbf{2 4}$ & 10.43 & 7.65 \\
\hline & &
\end{tabular}

For 24 hours ahead forecast, the nRMSE is $10.43 \%$ and nMAE is $7.65 \%$. There is an increase in nRMSE of about $146 \%$ from 1 hour ahead to 24 hours ahead forecast and about $154 \%$ in terms of nMAE. Fig 5 shows the increase in nRMSE with an increase in the forecast horizon. Fig 6, shows the increase in the nMAE with the increase in the forecast horizon.

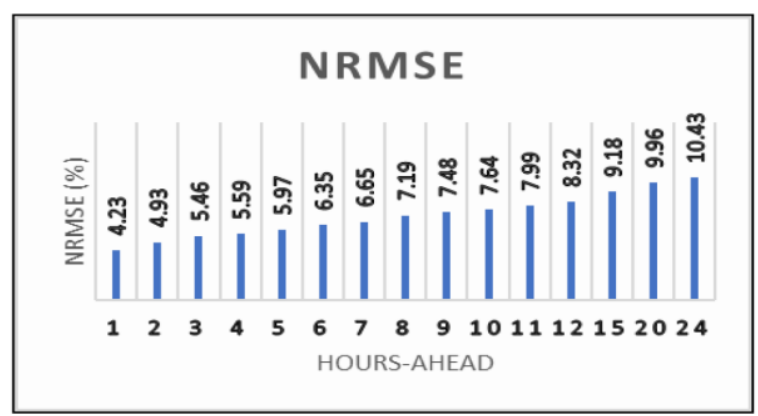

Fig. 5.1 to 24 hours ahead forecasting reuslts in terms of nRMSE.

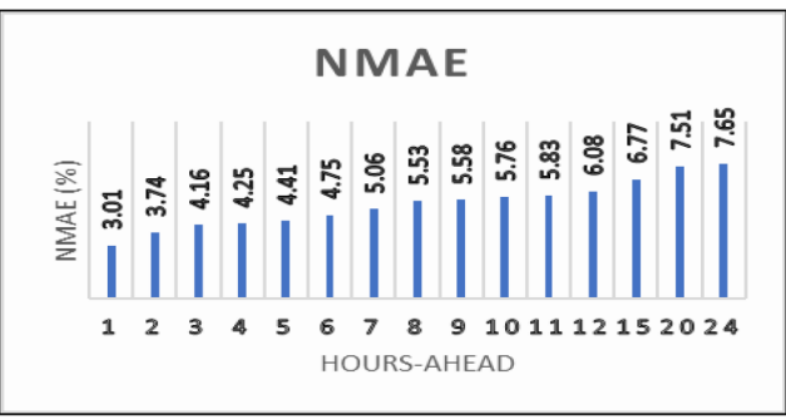

Fig. 6.1 to 24 hours ahead forecasting reuslts in terms of nMAE. 


\section{Conclusion}

This research paper presents an LSTM based RNN model to forecast short-term wind power. The model is trained on 9 months of data and tested on 3 months of the data. 24 lags are used to create the model with 1 hidden layer consisting of 50 hidden neurons. The input variables to the model are chosen after conducting a sensitivity analysis, testing the model with different input combinations. It is observed that surface pressure has a negative effect on the performance of the model. Temperature on the other hand has a positive effect, the addition of temperature increases the model's performance. The most accurate model is then used to forecast wind power with a forecast horizon from 1 hour ahead to 24 hours ahead.

In future, the LSTM-RNN model can be used to create ensemble and probabilistic wind power forecasts. Another area of interest would be to test the LSTM-RNN model in solar and load forecasting as well. Hybrid machine learning techniques as well as deep learning techniques can be explored in future to forecast wind power. Use of wind power forecast timeseries to improve unit commitment and economic dispatch as well as in predictive maintenance and power trading can be explored further.

\section{References}

[1] Anderson, TR., Hawkins E, and Jones PD. CO2, the greenhouse effect and global warming: From the pioneering work of Arrhenius and Callendar to today's Earth System Models. Endeavour, 2016. 40 3: 178-187.

[2] Saidur R. et al., A review on global wind energy policy. Renewable and Sustainable Energy Reviews, 2010. 14(7): $1744-1762$.

[3] Tsikalakis, AG., et al., Impact of wind power forecasting error bias on the economic operation of autonomous power systems. Wind Energy, 2009. 12(4): 315-331.

[4] Carlini, EM., et al. Physical and statistical downscaling for wind power forecasting. In: Proc. of 2016 International Symposium on Power Electronics, Electrical Drives, Automation and Motion (SPEEDAM). 2016.

[5] Tewari, S, Geyer CJ, Mohan N, A statistical model for wind power forecast error and its application to the estimation of penalties in liberalized markets. IEEE Transactions on Power Systems, 2011. 26(4): 2031-2039.

[6] Karakuş, O, Kuruoğlu EE, Altınkaya MA, One-day ahead wind speed/power prediction based on polynomial autoregressive model. IET Renewable Power Generation, 2017. 11(11): 1430-1439.

[7] Cao, Y, et al. Wind power ultra-short-term forecasting method combined with pattern-matching and ARMA-model. In: Proc. of 2013 IEEE Grenoble Conference, 2013.

[8] Kavasseri, RG. and Seetharaman K. Day-ahead wind speed forecasting using f-ARIMA models. Renewable Energy, 2009. 34(5): 1388-1393.

[9] Nayak, AK, et al. ARIMA based statistical approach to predict wind power ramps. In: Proc. of 2015 IEEE Power \& Energy Society General Meeting. 2015.

[10] Mao, Y. and W. Shaoshuai. A review of wind power forecasting \& prediction. In: Proc. of 2016 International Conference on Probabilistic Methods Applied to Power Systems (PMAPS). 2016.

[11] Kariniotakis, GN., Stavrakakis GS, and Nogaret EF, Wind power forecasting using advanced neural networks models. IEEE Transactions on Energy Conversion, 1996. 11(4): 762-767.

[12] Catalao, JPS, Pousinho HMI, and Mendes VMF. An artificial neural network approach for short-term wind power forecasting in Portugal. In: Proc. of 2009 15th International Conference on Intelligent System Applications to Power Systems. 2009.

[13] Ferreira, AA., et al. Investigating the use of Reservoir Computing for forecasting the hourly wind speed in short -term. In: Proc. of 2008 IEEE International Joint Conference on Neural Networks (IEEE World Congress on Computational Intelligence). 2008.

[14] Senjyu, T., et al. Application of recurrent neural network to long-term-ahead generating power forecasting for wind power generator. In: Proc. of 2006 IEEE PES Power Systems Conference and Exposition. 2006.

[15] Shi, Z., H. Liang, and V. Dinavahi, Direct Interval Forecast of Uncertain Wind Power Based on Recurrent Neural Networks. IEEE Transactions on Sustainable Energy, 2017: p. 1-1.

[16] Barbounis, T.G., et al., Long-term wind speed and power forecasting using local recurrent neural network models. IEEE Transactions on Energy Conversion, 2006. 21(1): p. 273-284.

[17] Selvin, S., et al. Stock price prediction using LSTM, RNN and CNN-sliding window model. In: Proc. of 2017 International Conference on Advances in Computing, Communications and Informatics (ICACCI). 2017.

[18] Zhang, Y. et al. A LSTM-RNN method for the lithuim-ion battery remaining useful life prediction. In: Proc. of 2017 Prognostics and System Health Management Conference (PHM-Harbin). 2017. 
[19] Nahid, MMH, Purkaystha B, and Islam MS. Bengali speech recognition: A double layered LSTM-RNN approach. In: Proc. of 2017 20th International Conference of Computer and Information Technology (ICCIT). 2017.

[20] Messina, R, Louradour J. Segmentation-free handwritten Chinese text recognition with LSTM-RNN. In: Proc. of 2015 13th International Conference on Document Analysis and Recognition (ICDAR). 2015.

[21] Li, Y., H. Wu, and H. Liu, Multi-step wind speed forecasting using EWT decomposition, LSTM principal computing, RELM subordinate computing and IEWT reconstruction. Energy Conversion and Management, 2018. 167: p. 203-219.

[22] Squartini, S., A. Hussain, and F. Piazza. Preprocessing based solution for the vanishing gradient problem in recurrent neural networks. in Circuits and Systems, 2003. ISCAS '03. Proceedings of the 2003 International Symposium on. 2003. 